\title{
Simultaneous Information and Energy Transmission: A Finite Block-Length Analysis
}

\author{
Samir M. Perlaza, Ali Tajer, and H. Vincent Poor
}

\begin{abstract}
In this paper, a non-asymptotic analysis of the fundamental limits of simultaneous information and energy transmission (SIET) is presented. The notion of the informationenergy capacity region, i.e., the largest set of simultaneously achievable information and energy rates, is revisited in a context in which transmissions occur within a finite number of channel uses and strictly positive decoding error probability (DEP) and energy shortage probability (ESP) are tolerated. The focus is on the case of one transmitter, one information receiver and one energy harvester communicating through binary symmetric memoryless channels. In this case, some outer bounds on the information transmission rate and the energy transmission rate are presented. More specifically, given a finite block-length, a DEP, and an ESP, four scenarios arise depending on whether an average or maximal probability constraint is imposed on the DEP and the ESP. For each scenario, the limits on the information rate and energy rate beyond which a transmission is no longer possible are presented (impossibility results). These results reveal the competitive interaction between the information transmission and energy transmission tasks identifying a certain regime in which increasing the information rate necessarily implies decreasing the energy rate and vice versa.
\end{abstract}

Index Terms-Information and Energy Transmission, Information and Power Transfer, Finite Block-Length Regime.

\section{INTRODUCTION}

Simultaneous information and energy transmission (SIET) refers to a communication system in which a set of transmitters aim to simultaneously carry on two tasks: information transmission to a set of information receivers (IRs); and energy transmission to a set of energy harvesters (EHs). The performance of SIET is often measured by the information and energy transmission rates that can be simultaneously achieved under certain reliability constraints. Reliability can be measured by two metrics: decoding error probability (DEP); and energy shortage probability (ESP). The fundamental limits of SIET consist of the largest set of information and energy rate tuples that can be simultaneously achieved. This set is

Samir M. Perlaza (samir.perlaza@inria.fr) is with the CITI Laboratory, a joint laboratory between the Institut National de Recherche en Informatique et en Automatique (INRIA), the Université de Lyon and the Institut National de Sciences Apliquées (INSA) de Lyon. 6 Av. des Arts 69621 Villeurbanne, France. He is also with the Department of Electrical Engineering at Princeton University, Princeton, NJ 08544 USA.

Ali Tajer is with the Department of Electrical, Computer, and Systems Engineering, Rensselaer Polytechnic Institute, Troy, NY 12180.

H. Vincent Poor (poor@princeton.edu) is with the Department of Electrical Engineering at Princeton University, Princeton, NJ 08544 USA.

This research was supported in part by the European Commission under Marie Skłodowska-Curie Individual Fellowship No. 659316 and EuroMediterranean Cooperation ERA-NET project COM-MED, and in part by the U.S. National Science Foundation under Grants CNS-1702808 and ECCS1647198. often referred to as the information-energy capacity region [1]. Traditionally, information-energy capacity regions are calculated subject to the fact that both DEP and ESP must be arbitrarily close to zero. This strong reliability constraint leads to the unavoidable use of infinitely long communication blocks and thus, these fundamental limits are meaningful only under the assumption that the communication might last a very long time. Within this context, the case of one transmitter and one co-located IR and EH is studied in [2], [3], and [4]. The multiuser case is studied in [5], [6], [7], [8], [9], [10], [11], [12], [13], [14], [15], and references therein. Despite the existing literature, these approaches quickly lose relevance in scenarios in which communications must occur within a short period, e.g., the Internet of things.

More relevant fundamental limits, from an engineering perspective, are those that take into account that SIET occurs within a finite number of channel uses and the system tolerates strictly positive DEP and ESP. This paper introduces this novel approach building upon the existing results on the fundamental limits of information transmission in the non-asymptotic block-length regime (c.f. [16] and [17]). The main result is the characterization of the information-energy capacity region of an SIET system with one transmitter, one IR, and one EH communicating over binary symmetric memoryless channels. More specifically, for a given number of channel uses and the biggest DEP and ESP that can be tolerated, the largest set of simultaneously achievable information and energy rates is characterized. The trade-off between information rate and energy rate, first reported in [2] and [3] in the asymptotic regime, is studied in the non-asymptotic regime. The system parameters for which information and energy transmission are conflicting tasks are also characterized. These results reveal the central role of the ESP in both the information transmission rate and the energy transmission rate.

The paper is organized as follows. Sec. II formulates the problem and introduces the notion of the information-energy capacity region in the non-asymptotic block-length regime. Sec. III focuses on the case of binary symmetric memoryless channels and presents the main results. Sec. IV concludes this work.

\section{SYSTEM MODEL}

Consider a three-party communication system in which a transmitter aims at simultaneously sending information to an IR and energy to an EH through a noisy communication medium. Such a system can be modeled by a random transformation $\left(\mathcal{X}, \mathcal{Y} \times \mathcal{Z}, P_{Y Z \mid X}\right)$ from an input alphabet $\mathcal{X}$ to an 
output alphabet $\mathcal{Y} \times \mathcal{Z}$ consisting of a transition probability kernel $P_{Y Z \mid X}$. That is, given an input $x \in \mathcal{X}$, the output $(y, z) \in \mathcal{Y} \times \mathcal{Z}$ is observed with probability $P_{Y Z \mid X}(y, z \mid x)$. In the following, the noisy communication medium is represented by a memoryless channel. A memoryless channel is a random transformation

$$
\left(\mathcal{X}^{n}, \mathcal{Y}^{n} \times \mathcal{Z}^{n}, P_{\boldsymbol{Y} \boldsymbol{Z} \mid \boldsymbol{X}}\right),
$$

where $n \in \mathbb{N}$ is the block length and $\boldsymbol{Y}=\left(Y_{1}, Y_{2}, \ldots, Y_{n}\right) \in$ $\mathcal{Y}^{n}, \quad \boldsymbol{Z}=\left(Z_{1}, Z_{2}, \ldots, Z_{n}\right) \in \mathcal{Z}^{n}$, and $\boldsymbol{X}=$ $\left(X_{1}, X_{2}, \ldots, X_{n}\right) \in \mathcal{X}^{n}$ are $n$-dimensional vectors of random variables, such that given an input $\boldsymbol{x}=\left(x_{1}, x_{2}, \ldots, x_{n}\right)$, the output $\left(y_{1}, y_{2}, \ldots, y_{n}, z_{1}, z_{2}, \ldots, z_{n}\right)$ is observed with probability

$$
P_{\boldsymbol{Y} \boldsymbol{Z} \mid \boldsymbol{X}}(\boldsymbol{y}, \boldsymbol{z} \mid \boldsymbol{x})=\prod_{t=1}^{n} P_{Y Z \mid X}\left(y_{t}, z_{t} \mid x_{t}\right) .
$$

Within this context, two tasks are carried out by the transmitter: $(a)$ the information transmission task; and $(b)$ the energy transmission task.

\section{A. Information Transmission Task}

The message to be sent from the transmitter to the IR is a realization of a random variable that is uniformly distributed in $\{1,2, \ldots, M\}$, with $1 \leqslant M<\infty$. To carry out this task within $n$ channel uses, the transmitter uses an $(n, M)$-code.

Definition $1((n, M)$-code): An $(n, M)$-code for the random transformation in (1) is a system

$$
\left\{\left(\boldsymbol{u}(1), \mathcal{D}_{1}\right),\left(\boldsymbol{u}(2), \mathcal{D}_{2}\right), \ldots,\left(\boldsymbol{u}(M), \mathcal{D}_{M}\right)\right\},
$$

where for all $(i, j) \in\{1,2, \ldots, M\}^{2}$, with $i \neq j$,

$$
\begin{aligned}
& \boldsymbol{u}(i) \triangleq\left(u_{1}(i), u_{2}(i), \ldots, u_{n}(i)\right) \in \mathcal{X}^{n}, \\
& \mathcal{D}_{i} \cap \mathcal{D}_{j}=\emptyset ; \text { and } \\
& \mathcal{D}_{1} \cup \mathcal{D}_{2} \cup \ldots \cup \mathcal{D}_{M} \subseteq \mathcal{Y}^{n} .
\end{aligned}
$$

Given the system in (3), to transmit the message index $i$, the transmitter inputs the symbol $u_{t}(i)$ to the channel at time $t \in\{1,2, \ldots, n\}$. The IR observes at the end of channel use $t$, the output $y_{t}$. At the end of $n$ channel uses, the IR states that the symbol $i$ was transmitted if it satisfies the rule

$$
\left(y_{1}, y_{2}, \ldots, y_{n}\right) \in \mathcal{D}_{i} .
$$

The probability of error associated with the transmission of message index $i$, denoted by $\lambda_{i} \in[0,1]$, is

$$
\lambda_{i} \triangleq \operatorname{Pr}\left[\boldsymbol{Y} \in \mathcal{D}_{i}^{c} \mid \boldsymbol{X}=\boldsymbol{u}(i)\right],
$$

where the probability is with respect to the marginal $P_{\boldsymbol{Y} \mid \boldsymbol{X}}$ of the joint probability distribution in (2). The average probability of error, denoted by $\lambda$, is

$$
\lambda \triangleq \frac{1}{M} \sum_{m=1}^{M} \lambda_{m} .
$$

Given a parameter $\epsilon \in[0,1]$, information transmission is said to be reliably performed in two different senses: (a) the average decoding error probability is smaller than $\epsilon$, i.e., $\lambda<\epsilon$, or

(b) the maximum decoding error probability is smaller than $\epsilon$, i.e., for all $i \in\{1,2, \ldots, M\}, \lambda_{i}<\epsilon$.

An $(n, M)$-code that exclusively satisfies the first criterion is said to be an $(n, M, \epsilon)$-code with average decoding error probability. Alternatively, an $(n, M)$-code that satisfies both criteria is said to be an $(n, M, \epsilon)$-code with maximal decoding error probability.

\section{B. Energy Transmission Task}

Let $g: \mathcal{Z} \rightarrow \mathbb{R}_{+}$be a positive-valued function. The amount of energy delivered to the EH by the channel outputs $\boldsymbol{z}=$ $\left(z_{1}, z_{2}, \ldots, z_{n}\right)$, given by the function $B_{n}: \mathcal{Z}^{n} \rightarrow \mathbb{R}_{+}$, is

$$
B_{n}(\boldsymbol{z})=\sum_{t=1}^{n} g\left(z_{t}\right) \text {. }
$$

The objective of the transmitter is to ensure that a minimum amount of energy $b$ is harvested at the $\mathrm{EH}$ at the end of $n$ channel uses. An energy shortage is said to occur when the energy harvested at the $\mathrm{EH}$ is less than the minimum required at the end of the transmission. The probability of an energy shortage when transmitting the message index $i \in\{1,2, \ldots, M\}$, denoted by $\theta_{i}$, is

$$
\theta_{i} \triangleq \operatorname{Pr}\left[B_{n}(\boldsymbol{Z})<b \mid \boldsymbol{X}=\boldsymbol{u}(i)\right],
$$

where the probability is with respect to the marginal $P_{\boldsymbol{Z} \mid \boldsymbol{X}}$ of the joint probability distribution in (2). The average probability of energy shortage, denoted by $\theta$, is

$$
\theta \triangleq \frac{1}{M} \sum_{i=1}^{M} \theta_{i} .
$$

Given a parameter $\delta \in[0,1]$, energy transmission is said to be reliably performed in two different senses:

(c) the average energy shortage probability is smaller than $\delta$, i.e., $\theta<\delta$, or

(d) the maximum energy shortage probability is smaller than $\delta$, i.e., for all $i \in\{1,2, \ldots, M\}, \theta_{i}<\delta$.

An $(n, M, \epsilon)$-code that exclusively satisfies the first criterion is said to be an $(n, M, \epsilon, \delta, b)$-code with average energy shortage probability. Alternatively, an $(n, M, \epsilon)$-code that satisfies both criteria is said to be an $(n, M, \epsilon, \delta, b)$-code with maximal energy shortage probability.

\section{Fundamental Limits}

The non-asymptotic fundamental limits of SIET are described by the notion of the information-energy capacity region.

Definition 2 (Information-Energy Capacity Region): The information-energy capacity region $\mathcal{C} \in \mathbb{N}^{2} \times[0,1]^{2} \times \mathbb{R}$ of the random transformation in (1) is the set of all tuples $(n, M, \epsilon, \delta, b)$ for which there exists an $(n, M, \epsilon, \delta, b)$-code satisfying at least one of the reliability criterion pairs: $(a, c)$; $(a, d) ;(b, c) ;$ or $(b, d)$. 


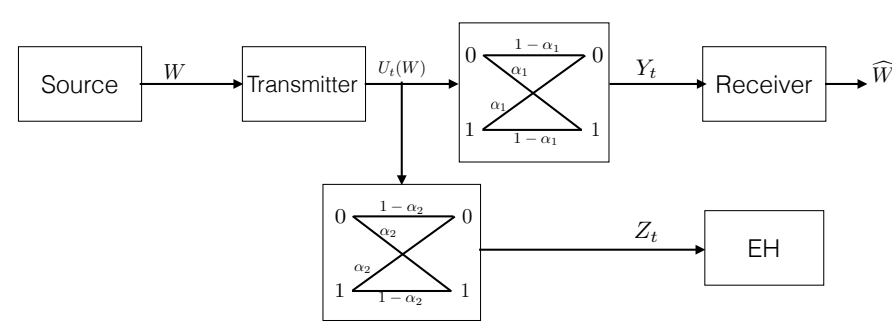

Fig. 1. A three-party communication system in which a transmitter aims at simultaneously sending information to an information receiver and energy to an energy harvester through binary symmetric channels.

The information-energy capacity region $\mathcal{C}$ in Definition 2 is a subset of $\mathbb{R}^{5}$ that is difficult to characterize. In this work, given fixed parameters $(n, \epsilon, \delta)$, the focus is on the subset $\mathcal{C}(n, \epsilon, \delta)$ that contains the pairs $(M, b) \in \mathbb{N} \times \mathbb{R}_{+}$ such that $(n, M, \epsilon, \delta, b) \in \mathcal{C}$. Therefore, if $(M, b) \in \mathcal{C}(n, \epsilon, \delta)$, then the information rate $\frac{\log _{2}(M)}{n}$ bits per channel use, and the energy rate $\frac{b}{n}$ energy units per channel use, are jointly achievable within $n$ channel uses with a (maximal and/or average) decoding error probability $\epsilon$ and (maximal and/or average) energy shortage probability $\delta$.

The subset $\mathcal{C}(n, \epsilon, \delta)$ is a subset of $\mathbb{R}^{2}$. In order to emphasize the reliability criterion, when needed, the informationenergy capacity region $\mathcal{C}(n, \epsilon, \delta)$ is often written as $\mathcal{C}_{(\mathrm{a}, \mathrm{c})}(n, \epsilon, \delta), \mathcal{C}_{(\mathrm{a}, \mathrm{d})}(n, \epsilon, \delta), \mathcal{C}_{(\mathrm{b}, \mathrm{c})}(n, \epsilon, \delta)$ or $\mathcal{C}_{(\mathrm{b}, \mathrm{d})}(n, \epsilon, \delta)$, respectively. A similar notation is used for the larger region $\mathcal{C}$.

\section{Memoryless Binary Symmetric Channels}

This section focuses on the case in which $\mathcal{X}=\mathcal{Y}=\mathcal{Z}=$ $\{0,1\}$ and the random transformation in (1) is such that for all $\boldsymbol{x} \in\{0,1\}^{n}$, the outputs $\boldsymbol{y} \in\{0,1\}^{n}$ and $\boldsymbol{z} \in\{0,1\}^{n}$ are observed with probability

$$
P_{\boldsymbol{Y} \boldsymbol{Z} \mid \boldsymbol{X}}(\boldsymbol{y}, \boldsymbol{z} \mid \boldsymbol{x})=\prod_{t=1}^{n} P_{Y \mid X}\left(y_{t} \mid x_{t}\right) P_{Z \mid X}\left(z_{t} \mid x_{t}\right),
$$

where for all $(x, y, z) \in\{0,1\}^{3}$,

$$
\begin{aligned}
& P_{Y \mid X}(y \mid x)=\alpha_{1} \mathbb{1}_{\{x \neq y\}}+\left(1-\alpha_{1}\right) \mathbb{1}_{\{x=y\}}, \\
& P_{Z \mid X}(z \mid x)=\alpha_{2} \mathbb{1}_{\{x \neq z\}}+\left(1-\alpha_{2}\right) \mathbb{1}_{\{x=z\}},
\end{aligned}
$$

and $\alpha_{1} \in\left[0, \frac{1}{2}\right)$ and $\alpha_{2} \in\left(0, \frac{1}{2}\right)$ are the crossover probabilities of the channel. This scenario is depicted by Figure 1. Let

$$
b_{0}=g(0) \geqslant 0 \text { and } b_{1}=g(1) \geqslant 0,
$$

be the energy harvested when the output of the channel at the EH is 0 and 1 , respectively. The case in which $b_{0}=b_{1}$ is trivial, since the harvested energy is always $n b_{1}=n b_{0}$ energy units, independently of the codebook. This implies that the information transmission task can be carried out without taking into account the energy transmission task. Hence, for avoiding the trivial cases, the following assumption is adopted without loss of generality:

$$
b_{1}<b_{0} .
$$

For all $\boldsymbol{z} \in\{0,1\}^{n}$, it follows that

$$
\begin{aligned}
B_{n}(\boldsymbol{z}) & =b_{0} N(0 \mid \boldsymbol{z})+b_{1} N(1 \mid \boldsymbol{z}) \\
& =\left(b_{0}-b_{1}\right) N(0 \mid \boldsymbol{z})+n b_{1},
\end{aligned}
$$

where $N(0 \mid \boldsymbol{z})$ and $N(1 \mid \boldsymbol{z})$ are the numbers of zeros and ones in the vector $\boldsymbol{z}$, respectively. Note that $B_{n}(\boldsymbol{z})$ is bounded for all $z \in \mathcal{Z}^{n}$, i.e.,

$$
n b_{1} \leqslant B_{n}(\boldsymbol{z}) \leqslant n b_{0} .
$$

The inequalities in (18) imply that there exists a case in which energy transmission might occur with zero (maximal or average) energy shortage probability at a given energy rate $\frac{b}{n} \leqslant b_{1}$. This is because the event $B_{n}(\boldsymbol{Z})<n b_{1}$ is observed with zero probability. Basically, transmitting any symbol either zero or one is indifferent from the energy transmission perspective. In this case, the information transmission task can be carried out independently of the energy transmission task given that $n b_{1}$ energy units can always be reliably transmitted in $n$ channel uses. Alternatively, any energy transmission rate $\frac{b}{n}>b_{0}$ cannot be achieved with an average or maximal energy shortage probability strictly smaller than one.

Given an $(n, M)$-code described by the system in (3), let the empirical probability distribution of the channel input symbols induced by the codeword $\boldsymbol{u}(i)$, denoted by $\bar{P}_{X}^{(i)}$, be such that

$$
\bar{P}_{X}^{(i)}(0)=1-\bar{P}_{X}^{(i)}(1)=\frac{1}{n} \sum_{t=1}^{n} \mathbb{1}_{\left\{u_{t}(i)=0\right\}},
$$

for all $i \in\{1,2, \ldots, M\}$. Let also the empirical distribution of the channel input symbols jointly induced by all codewords, denoted by $\bar{P}_{X}(0)$, be such that

$$
\bar{P}_{X}(0)=1-\bar{P}_{X}(1)=\frac{1}{n M} \sum_{i=1}^{M} \sum_{t=1}^{n} \mathbb{1}_{\left\{u_{t}(i)=0\right\}} .
$$

Using these empirical distributions some upper bounds can be obtained on both the energy and information transmission rates.

\section{A. An Outer Bound on the Energy Rate of $(n, M)$-Codes}

Define $Q: \mathbb{R} \rightarrow[0,1]$ as the complementary cumulative distribution function of the standard normal distribution, and define $Q^{-1}:(0,1) \rightarrow \mathbb{R}$ as the functional inverse of $Q$. Using this notation, the following proposition provides an outer bound on the number of energy units $b$ that can be reliably delivered by any given $(n, M)$-code with an average or maximal energy shortage probability $\delta \in(0,1)$.

Proposition 1: Consider an $(n, M, \epsilon, \delta, b)$-code described by the system in (3) for the random transformation in (11) satisfying (15). Then, subject to a maximal energy shortage probability constraint, it holds that for all $i \in\{1,2, \ldots, M\}$,

$$
\begin{aligned}
b & <n\left(\left(b_{0}-b_{1}\right)\left(\left(1-2 \alpha_{2}\right) \bar{P}_{X}^{(i)}(0)+\alpha_{2}\right)+b_{1}\right) \\
& -\sqrt{n\left(b_{0}-b_{1}\right)^{2} \alpha_{2}\left(1-\alpha_{2}\right)} Q^{-1}(\delta)+\mathcal{O}(1),
\end{aligned}
$$


and subject to an average energy shortage probability constraint, it holds that

$$
\begin{gathered}
b<n\left(\left(b_{0}-b_{1}\right)\left(\left(1-2 \alpha_{2}\right) \bar{P}_{X}(0)+\alpha_{2}\right)+b_{1}\right) \\
-\sqrt{n\left(b_{0}-b_{1}\right)^{2} \alpha_{2}\left(1-\alpha_{2}\right)} Q^{-1}(\delta)+\mathcal{O}(1) .
\end{gathered}
$$

Proof of Proposition 1: Note that an $(n, M, \epsilon, \delta, b)$-code with maximal energy shortage probability satisfies for all $i \in$ $\{1,2, \ldots, M\}$,

$$
\operatorname{Pr}\left[B_{n}(\boldsymbol{Z})<b \mid \boldsymbol{X}=\boldsymbol{u}(i)\right]<\delta,
$$

where the probability operator in (23) applies with respect to the marginal $P_{\boldsymbol{Z} \mid \boldsymbol{X}}$ of the joint distribution in (11). Note also that the random variable $B_{n}(\boldsymbol{Z})$ in (23) is the sum of $n$ independent binary random variables $\mathbb{1}_{\left\{Z_{t}=0\right\}}$, where $Z_{t}$ follows the distribution $P_{Z \mid X=u_{t}(i)}$. That is,

$\operatorname{Pr}\left[B_{n}(\boldsymbol{Z})<b \mid \boldsymbol{X}=\boldsymbol{u}(i)\right]=\operatorname{Pr}\left[\sum_{t=1}^{n} \mathbb{1}_{\left\{Z_{t}=0\right\}}<\left(\frac{b-n b_{1}}{b_{0}-b_{1}}\right)\right]$.

Hence, from the Berry-Essen theorem [18], it follows that for all $i \in\{1,2, \ldots, M\}$,

$$
\begin{aligned}
& \operatorname{Pr}\left[B_{n}(\boldsymbol{Z})<b \mid \boldsymbol{X}=\boldsymbol{u}(i)\right] \geqslant-\frac{\left(1-\alpha_{2}\right)^{2}+\alpha_{2}^{2}}{2 \sqrt{n \alpha_{2}\left(1-\alpha_{2}\right)}} \\
& +Q\left(\frac{n\left(\left(1-2 \alpha_{2}\right) \bar{P}_{X}^{(i)}(0)+\alpha_{2}\right)-\frac{b-n b_{1}}{b_{0}-b_{1}}}{\sqrt{n \alpha_{2}\left(1-\alpha_{2}\right)}}\right) .
\end{aligned}
$$

Thus, from (23) and (24), it follows that

$$
\begin{aligned}
b< & n\left(\left(b_{0}-b_{1}\right)\left(\left(1-2 \alpha_{2}\right) \bar{P}_{X}(0)+\alpha_{2}\right)+b_{1}\right) \\
& -\sqrt{n\left(b_{0}-b_{1}\right)^{2} \alpha_{2}\left(1-\alpha_{2}\right)} Q^{-1}\left(\delta+\frac{\left(1-\alpha_{2}\right)^{2}+\alpha_{2}^{2}}{2 \sqrt{n \alpha_{2}\left(1-\alpha_{2}\right)}}\right) \\
< & n\left(\left(b_{0}-b_{1}\right)\left(\left(1-2 \alpha_{2}\right) \bar{P}_{X}(0)+\alpha_{2}\right)+b_{1}\right) \\
& -\sqrt{n\left(b_{0}-b_{1}\right)^{2} \alpha_{2}\left(1-\alpha_{2}\right)} Q^{-1}(\delta)+\mathcal{O}(1),
\end{aligned}
$$

where the last inequality in (25) follows from a Taylor approximation of the function $Q^{-1}$. A similar procedure can be performed to prove (22). This completes the proof.

For fixed parameters $\left(n, \alpha_{2}, \delta, b_{0}, b_{1}\right)$, let $\rho^{*}: \mathbb{R}_{+} \rightarrow \mathbb{R}_{+}$ be defined as

$$
\rho^{*}(b)=\min \left(1, \rho^{+}(b)\right),
$$

with

$$
\begin{aligned}
& \rho^{+}(b)= \\
& \frac{b-n\left(\left(1-\alpha_{2}\right) b_{1}+\alpha_{2} b_{0}\right)}{n\left(b_{0}-b_{1}\right)\left(1-2 \alpha_{2}\right)}+\frac{\sqrt{\alpha_{2}\left(1-\alpha_{2}\right)}}{\sqrt{n}\left(1-2 \alpha_{2}\right)} Q^{-1}(\delta)+\mathcal{O}\left(\frac{1}{n}\right) \text {. }
\end{aligned}
$$

Using this notation, the following corollary follows immediately from Proposition 1.

Corollary 1: Consider an $(n, M, \epsilon, \delta, b)$-code described by the system in (3) for the random transformation in (11) satisfying (15). Then, subject to a maximal energy shortage probability constraint, for all $i \in\{1,2, \ldots, M\}$, the empirical input distribution $\bar{P}_{X}^{(i)}$ satisfies

$$
\bar{P}_{X}^{(i)}(0)>\rho^{*}(b)
$$

and subject to an average energy shortage probability constraint, it follows that

$$
\bar{P}_{X}(0)>\rho^{*}(b)
$$

where $\rho^{*}(b)$ is defined in (26).

Corollary 1 leads to interesting conclusions by noticing that $\rho^{*}(b)$ is a lower bound on the fraction of zeros in each codeword (maximal energy shortage probability) or the fraction of zeros among all codewords (average energy shortage probability) when energy is transmitted at an average energy rate $\frac{b}{n}$. This is natural from the perspective of the assumption in (15), which implies that the symbol zero carries more energy that the symbol one.

Note that the input distribution that achieves the largest information transmission rate is the uniform distribution [16]. That is, $\bar{P}_{X}(0)=1-\bar{P}_{X}(1)=\frac{1}{2}$. Hence, Proposition 1 provides an outer bound on the energy rate that can be transmitted by an $(n, M, \epsilon, \delta, b)$-code that possesses an empirical input distribution that is information-rate optimal. The following corollary describes this observation.

Corollary 2: Consider an $(n, M, \epsilon, \delta, b)$-code for the random transformation in (11) satisfying (15). Assume that such a code exhibits an information-rate optimal empirical distribution. Then, it follows that $b<\underline{b}(n, \delta)$, with $\underline{b}: \mathbb{N} \times[0,1] \rightarrow \mathbb{R}$, such that

$$
\begin{aligned}
\underline{b}(n, \delta)= & n\left(\frac{b_{0}+b_{1}}{2}\right)-\sqrt{n\left(b_{0}-b_{1}\right)^{2} \alpha_{2}\left(1-\alpha_{2}\right)} Q^{-1}(\delta) \\
& +\mathcal{O}(1) .
\end{aligned}
$$

Essentially, Corollary 2 determines a threshold on the number of energy units $b$ beyond which an $(n, M, \epsilon, \delta, b)$-code, if it exists, exhibits a conflict between the energy transmission task and the information transmission task. More specifically, if there exists an $(n, M, \epsilon, \delta, b)$-code whose energy transmission rate $b$ is beyond the threshold $\underline{b}$, it exhibits an empirical input distribution for which $\bar{P}_{X}(0)>\bar{P}_{X}(1)$. This implies than a zero is transmitted more often than a one, which is not information-rate optimal.

Proposition 1 also provides an outer bound on the largest energy rate that can be transmitted by any $(n, M, \epsilon)$-code. Note that the largest energy-transmission rate is achieved by a zero information-rate code whose codewords contain only zeros, i.e., $\bar{P}_{X}(0)=1-\bar{P}_{X}(1)=1$. The following corollary describes this observation.

Corollary 3: Consider an $(n, M, \epsilon, \delta, b)$-code for the random transformation in (11) satisfying (15). Then, it follows that: $b<\bar{b}(n, \delta)$, with $\bar{b}: \mathbb{N} \times[0,1] \rightarrow \mathbb{R}$, such that

$$
\begin{aligned}
\bar{b}(n, \delta)= & n\left(\left(1-\alpha_{2}\right) b_{0}+\alpha_{2} b_{1}\right) \\
& -\sqrt{n\left(b_{0}-b_{1}\right)^{2} \alpha_{2}\left(1-\alpha_{2}\right)} Q^{-1}(\delta)+\mathcal{O}(1)
\end{aligned}
$$


Finally, from Corollary 2 and Corollary 3, it might be expected that the energy transmission task and the information transmission task exhibit a conflicting interaction. The following section explores this particular interaction.

\section{B. An Outer Bound on the Information Rate of $(n, M)$-Codes}

Given an $(n, M, \epsilon, \delta, b)$-code, the following proposition describes a bound on $M$. This bound does not take into account the decoding error probability and thus, it might be loose in some cases. However, it plays an important role when $\underline{b}(n, \delta)<b<\bar{b}(n, \delta)$.

Proposition 2: Consider an $(n, M, \epsilon, \delta, b)$-code for the random transformation in (11) satisfying (15). Then, subject to a maximal energy shortage probability constraint, it holds that

$$
M \leqslant\left(\begin{array}{c}
n \\
\left\lceil n \rho^{*}(b)\right\rceil
\end{array}\right) 2^{\left(n-\left\lceil n \rho^{*}(b)\right\rceil\right)},
$$

and subject to an average energy shortage probability constraint, it follows that

$$
M \leqslant\left(\begin{array}{c}
M n \\
\left\lceil M n \rho^{*}(b)\right\rceil
\end{array}\right) 2^{\left(M n-\left\lceil M n \rho^{*}(b)\right\rceil\right)},
$$

where $\rho^{*}(b)$ is defined by (26).

Proof of Proposition 2: Corollary 1 provides an approximation to the minimum number of zeros in each codeword in any given $(n, M, \epsilon, \delta, b)$-code with maximal probability of energy shortage. That is, for all $i \in\{1,2, \ldots, M\}$ if follows that

$$
N(0 \mid \boldsymbol{u}(i)) \geqslant\left\lceil n \rho^{*}(b)\right\rceil .
$$

This immediately provides the upper-bound on $M$ given that all codewords must contain at least $\left\lceil n \rho^{*}(b)\right\rceil$ zeros. Hence, the right-hand side of (31) is the maximum number of codewords of length $n$ for which at least $\left[n \rho^{*}(b)\right]$ symbols are zeros. The same argument can be used to prove the fixed-point equation in (32). This completes the proof.

Note that $\rho^{*}(b)$ is monotonically increasing with the energy rate $b$. Interestingly, when $\rho^{*}(b) \in\left(\frac{1}{2}, 1\right]$, the right-hand sides of (31) and (32) are monotonically decreasing with $b$. This highlights the existing trade-off between the information transmission task and the energy transmission task. That is, in the regime in which $\rho^{*}(b) \in\left(\frac{1}{2}, 1\right]$, increasing the energy rate would necessarily imply decreasing the information rate.

\section{CONCLUSIONS}

In this paper, the fundamental limits of SIET have been studied under the assumption that the transmission occurs during a finite number of channel uses at the expense of strictly positive DEP and ESP. From this perspective, a non-asymptotic fundamental limit has been introduced: the information-energy capacity region, that is, the largest set of jointly achievable energy and information rates. The focus has been on the case of one transmitter, one IR and one EH communicating via binary symmetric memoryless channels. In this case, given a finite block-length, a DEP, and an ESP, four scenarios have been observed depending on whether an average or maximal probability constraint is imposed on the
DEP and the ESP. For each scenario, the limits on the information rate and energy rate beyond which a transmission is no longer possible have been characterized. These results have revealed the competitive interaction between the information transmission task and energy transmission task. In particular, a certain regime in which increasing the information rate necessarily implies decreasing the energy rate and vice versa has been identified.

\section{REFERENCES}

[1] S. Belhadj Amor and S. M. Perlaza, "Fundamental limits of simultaneous energy and information transmission," in Proc. International Symposium on Telecommunications, Thessaloniki, Greece, May 2016.

[2] L. R. Varshney, "Transporting information and energy simultaneously," in Proc. IEEE International Symposium on Information Theory, Toronto, ON, Canada, Jul. 2008, pp. 1612-1616.

[3] P. Grover and A. Sahai, "Shannon meets Tesla: Wireless information and power transfer," in Proc. IEEE International Symposium on Information Theory, Jun. 2010, pp. 2363-2367.

[4] M. Varasteh, B. Rassouli, and B. Clerckx, "Wireless information and power transfer over an AWGN channel: Nonlinearity and asymmetric Gaussian signaling," in Proc. IEEE Information Theory Workshop, Kaohsiung, Taiwan, Nov. 2017.

[5] S. Belhadj Amor, S. M. Perlaza, I. Krikidis, and H. V. Poor, "Feedback enhances simultaneous wireless information and energy transmission in multiple access channels," IEEE Trans. Inf. Theory, vol. 63, no. 8, pp. 5244-5265, Aug. 2017.

[6] J. Park and B. Clerckx, "Joint wireless information and energy transfer in a two-user MIMO interference channel," IEEE Trans. Wireless Commun., vol. 12, no. 8, pp. 4210-4221, Aug. 2013.

[7] A. M. Fouladgar and O. Simeone, "On the transfer of information and energy in multi-user systems," IEEE Communications Letters, vol. 16, no. 11, pp. 1733-1736, Nov. 2012.

[8] S. Ulukus, A. Yener, E. Erkip, O. Simeone, M. Zorzi, P. Grover, and K. Huang, "Energy harvesting wireless communications: A review of recent advances," IEEE Journal on Selected Areas in Communications, vol. 33, no. 3, pp. 360-381, Mar. 2015.

[9] P. Popovski, A. M. Fouladgar, and O. Simeone, "Interactive joint transfer of energy and information," IEEE Transactions on Communications, vol. 61, no. 5, pp. 2086-2097, May 2013.

[10] R. Zhang and C. K. Ho, "MIMO broadcasting for simultaneous wireless information and power transfer," IEEE Transactions on Wireless Communications, vol. 12, no. 5, pp. 1989-2001, May 2013.

[11] M. Gastpar, "Gaussian multiple-access channels under received-power constraints," in Proc. IEEE Information Theory Workshop, San Antonio, TX, USA, Mar. 2004, pp. 452-457.

[12] I. Krikidis, S. Timotheou, S. Nikolaou, G. Zheng, D. W. K. Ng, and R. Schober, "Simultaneous wireless information and power transfer in modern communication systems," IEEE Communications Magazine, vol. 52, pp. 104-110, Nov. 2014.

[13] Z. Ding, S. M. Perlaza, I. Esnaola, and H. V. Poor, "Power allocation strategies in energy harvesting wireless cooperative networks," IEEE Transactions on Wireless Communications, vol. 13, no. 2, pp. 846-860, Feb. 2014.

[14] N. Khalfet and S. M. Perlaza, "Simultaneous information and energy transmission in Gaussian interference channels," in Proc. 2018 International Zurich Seminar on Information and Communication, Zurich, Switzerland, Feb. 2017.

[15] — "Simultaneous information and energy transmission in Gaussian interference channels with feedback," in Proc. 55th Annual Allerton Conference on Communications, Control, and Computing, Monticello, IL, USA, Oct. 2017.

[16] Y. Polyanskiy, "Channel coding: Non-asymptotic fundamental limits," Ph.D. dissertation, Electrical Engineering, Princeton University, Princeton, NJ, USA, Nov. 2010.

[17] Y. Polyanskiy, H. V. Poor, and S. Verdú, "Channel coding rate in the finite blocklength regime," IEEE Trans. Inf. Theory, vol. 56, no. 5, pp. 2307-2359, May 2010.

[18] W. Feller, An Introduction to Probability Theory and Its Application, 2nd ed. New York, NY: John Wiley and Sons, 1971, vol. 2. 